\title{
Erratum to: Fluorescence Quenching of Humic Acid by Coated Metallic Silver Particles
}

\author{
Guocheng Zhu ${ }^{1}$ • Jun Yin ${ }^{2}$
}

Published online: 10 April 2017

(C) Springer Science+Business Media New York 2017

Erratum to: J Fluoresc.

DOI 10.1007/s10895-016-2012-z

The original article was corrected. Table 1 previously contained an incorrect header "ND 90 (nm)" which should read "D $90(\mathrm{~nm})$ ".

The online version of the original article can be found at http://dx.doi. org/10.1007/s10895-016-2012-z

Guocheng Zhu

zhuguoc@hnust.edu.cn; zgc945ahhn@163.com

1 Hunan Provincal Key Laboratory of Shale Gas Resource Utilization, Hunan University of Science and Technology, Xiangtan 411201,

China

2 Department of Civil and Environmental Engineering, University of Missouri, Columbia, MO 65211, USA 